\title{
PREDICCIÓN DE LA IRRADIANCIA A PARTIR DE DATOS DE SATÉLITE MEDIANTE DEEP LEARNING
}

\section{Resumen}

El conocimiento con antelación de la irradiancia solar en la superfície en una ubicación determinada presenta diversas utilidades para las plantas fotovoltaicas, como la participación más ventajosa en el mercado eléctrico o las menores necesidades de almacenamiento para ello. En este trabajo se estudian diferentes alternativas para la estimación de la irradiancia a posteriori y se propone una red neuronal artificial que, utilizando esta estimación, predice la irradiancia solar futura con una hora de antelación.
\end{abstract}

J. Pérez

Dep. de Ingeniería y Ciencia de los computadores, perezsolerj@gmail.com

\begin{abstract}
J. Segarra-Tamarit, H. Beltran, C. Ariño, J.C. Alfonso-Gil, A. Attanasio, E. Pérez
Dep. de Ingeniería de Sistemas Industriales y Diseño, Universitat Jaume I, Castelló de la Plana, España
\end{abstract}

Palabras clave: Predicción, Irradiancia solar, redes neuronales, deep learning, MSGCPP, MACC-RAD

\section{INTRODUCCIÓN}

El crecimiento exponencial experimentado de forma prácticamente sostenida durante los últimos 10 años por el sector de la energía fotovoltaica le ha llevado a contar con más de $300 \mathrm{GW}$ de potencia instalada a cierre del año 2016, y con una previsión de alcanzar cerca de $370 \mathrm{GW}$ a finales de 2017[1]. Esto hace que a día de hoy la fotovoltaica sea la principal tecnología de producción de energía eléctrica a nivel de nuevas instalaciones a escala mundial[2].

La continua e imparable penetración de la fotovoltaica en los sistemas eléctricos de los distintos países implica un reto asociado a la estabilidad de estos sistemas [3] ya que, cabe recordar, la fotovoltaica es una tecnología cuya producción es inherentemente intermitente. Así, la estocástica evolución de las nubes a lo largo del día comporta que las plantas fotovoltaicas no puedan garantizar una producción determinada en cualquier momento del día. Esto ha venido dificultando tanto la gestión de la red por parte de los operadores como la participación de estas plantas en los mercados de compraventa de electricidad de los distintos países.

Por otro lado, la irrupción en el mercado eléctrico con unos precios cada vez más competitivos de varias tecnologías de almacenamiento de energía, principalmente baterías de iones de litio y de sulfuro de sodio, ha abierto la puerta a la aparición de proyectos fotovoltaicos híbridos que incorporan baterías[4]. Estas nuevas plantas presentan curvas de producción mucho más controladas ya que las baterías ejercen el rol de buffer energético. Por tanto, las baterías permiten suplir las limitaciones de la fotovoltaica a nivel de predictibilidad pero siguen siendo un elemento caro que aumenta bastante el coste de la instalación, reduciendo su rentabilidad. Además, las distintas clases de batería presentan todavía vidas útiles asociadas al ciclado relativamente limitadas [5], [6], [7] y [8], cosa que implica la necesidad de sustituciones a lo largo de la vida de la planta.

La posibilidad de predecir la irradiancia disponible en una ubicación se antoja en este sentido un factor fundamental ya no solo para mejorar la posible venta de electricidad con mayor fiabilidad por parte de las plantas fotovoltaicas al mercado eléctrico, sino también para poder optimizar el funcionamiento de la planta incluyendo como parámetro de operación la minimización del envejecimiento de las baterías.

En este artículo se presenta una metodología que permite predecir con una hora de antelación cual será la irradiancia en una ubicación determinada con un error sistemático de 0.01 y una desviación típica de 0.18 .

\section{IRRADIANCIA A PARTIR DE IMÁGENES DE SATÉLITE}

Una de las metodologías más utilizadas para realizar estimaciones de irradiancia solar sobre la superficie terrestre se basa en el uso de imagenes de satélite, que a nivel europeo proceden del sistema de satélites Meteosat de segunda generación $M S G$. No obstante, existen varias plataformas o bases de datos que ofrecen distintos tipos de estimación, para las distintas ubicaciones y para cada momento específico, obtenidas mediante cálculos y algoritmos propios a partir de dichas imágenes de satélites. En este estudio se han utilizado y evaluado los datos producidos por dos de es- 
tas plataformas: $M S G C P P$ [9], desarrollada por el Koninklijk Nederlands Meteorologisch Instituut, y $M A C C-R A D$ [10], promovida por la Comisión Europea y que forma parte del programa para la observación de la tierra, Copernicus.

Por lo que respecta a $M S G C P P$, ésta utiliza para obtener la irradiancia un algoritmo basado en las propiedades físicas de las nubes identificadas en las imágenes de satélite. De este modo, permite descargar datos de diferentes parámetros (tales como temperatura y altura de las nubes, precipitaciones o radiación horizontal) estimados con un periodo de muestreo de 15 minutos y una resolución espacial de $3 \times 3 \mathrm{~km}^{2}$.

En cambio, dentro de los múltiples datos relativos a diferentes parámetros relacionados con el clima que Copernicus pone a disposición de los usuarios, el servicio de $M A C C$-RAD proporciona series de datos de radiación horizontal en cualquier lugar de Europa con un periodo de muestreo de hasta un minuto. Los datos se calculan interpolando para la posición solicitada y los valores disponibles más recientes son de dos días antes de la fecha actual.

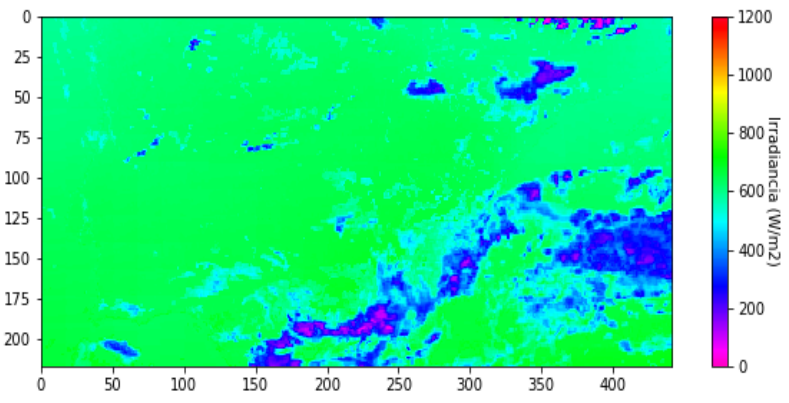

Figura 1: Mapa de irradiancias a partir de MSGCPP

Gracias a los valores de irradiancia proporcionados por estos modelos, se puede formar una matriz que represente la irradiancia de una zona como la representada en la figura 1 . De este modo, más allá de la información relativa al punto de estudio se pueden conocer valores de irradiancia en zonas aledañas y, si se toma una sucesión de imágenes, se puede observar la evolución de las zonas de baja irradiancia causadas por las nubes.

\section{COMPARACIÓN MSGCPP vs MACC-RAD}

Las bases de datos introducidas ofrecen estimaciones de los valores de irradiancia puntuales y discretos que deben ser contrastados en todo caso con medidas reales para valorar su fiabilidad y utilidad. En [11] los autores estudian el error existente entre varios de los métodos de cálculo disponibles en Europa y concluyen que el error cuadrático medio (RMSE) puede estar entre un $3 \%$ y un $6 \%$ cuando se agrupan los valores de manera mensual. Sin embargo, este error aumenta al reducir el periodo de los datos a nivel horario o diario.

Por lo que respecta a las bases de datos consideradas, la figura 2 muestra los valores de estimaciones realizadas para varios días consecutivos del año 2016. Se puede apreciar como ambos modelos son capaces de detectar la presencia de nubes y ofrecen valores de irradiancia similares a los reales. También se comprueba como para las primeras y últimas horas del día, MSGCPP no ofrece valores (debido a la inexistencia de imágenes de satélite). Sin embargo este modelo se aproxima más a los valores reales.

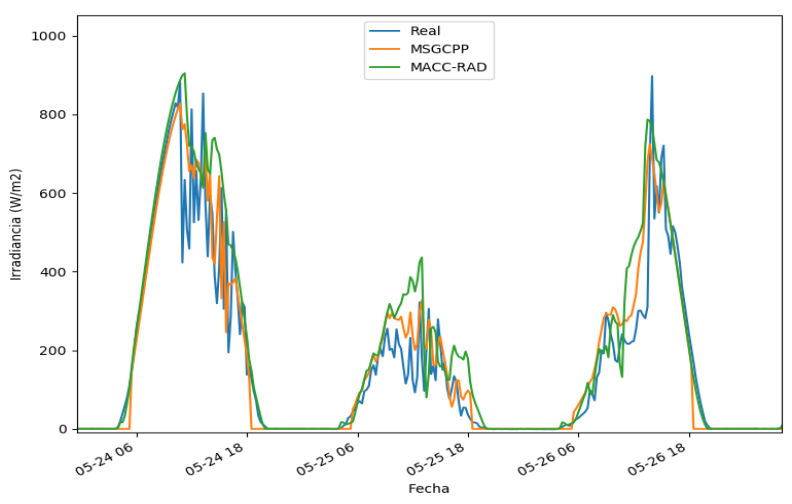

Figura 2: Comparación de datos para tres días

Se ha calculado con una frecuencia mensual el error entre los dos modelos y los valores reales. Esto se ha hecho como en [11], separando el $R M$ $S E$ en una componente sistemática (Bias) y una desviación estándar $(S D E)$ de acuerdo a la ecuación 1 .

$$
R M S E^{2}=S D E^{2}+\text { Bias }^{2}
$$

El RMSE normalizado se calcula a partir de las medidas de irradiancia real $\left(G_{\text {real }}\right)$ y de la irradiancia estimada $\left(G_{e s t}\right)$, que se quiere analizar, utilizando la ecuación 2

$$
R M S E=\sqrt{\frac{\sum\left(G_{\text {est }}-G_{\text {real }}\right)^{2}}{N \cdot \bar{G}_{\text {real }}^{2}}}
$$

donde $N$ es el número de muestras y $\bar{G}_{\text {real }}$ es el valor medio de irradiancia real. Por otra parte, el Bias normalizado se calcula utilizando la ecuación 3

$$
\text { Bias }=\frac{1}{N} \sum\left(\frac{G_{e s t}-G_{\text {real }}}{\bar{G}_{\text {real }}}\right)
$$


El resultado del error obtenido entre cada uno de los modelos y la realidad para 16 meses consecutivos, entre enero de 2016 y abril de 2017, se muestra en la figura 3. Se comprueba cómo el error mensual de $M S G C P P$ es menor, ya que sus puntos están más próximos al 0 tanto en desviación estándar como en desviación sistemática.

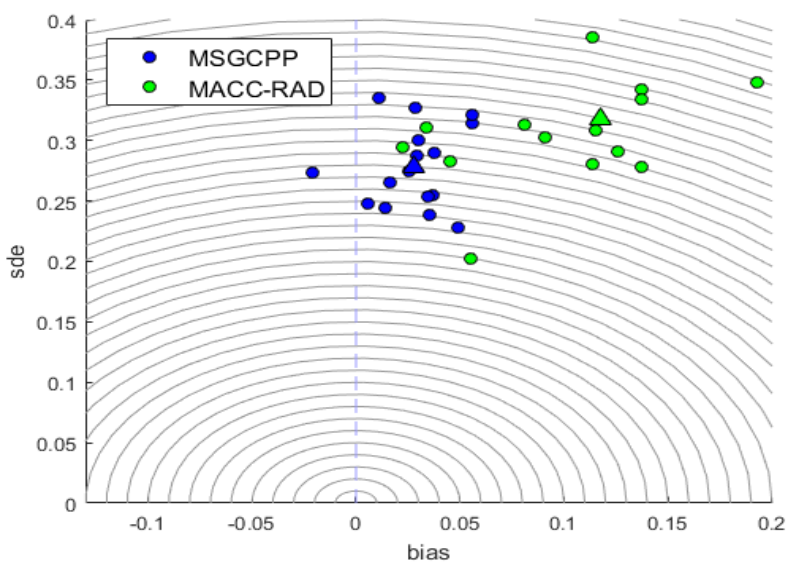

Figura 3: Representación de los errores mensuales de $M S G C P P$ y $M A C C-R A D$

\section{PREDICCIÓN DE IRRADIANCIA MEDIANTE DEEP LEARNING}

El campo de las redes neuronales artificiales, y más concretamente el conocido como Deep learning, presentado en [12], está revolucionando muchos campos de investigación que se encontraban estancados obteniendo resultados muy por encima del estado del arte hasta el momento. Esto es especialmente cierto en el campo de reconocimiento de imágenes, donde las redes neuronales se han mostrado muy efectivas como en [13], pero también en otros campos de aplicación tan diversos como el descubrimiento de partículas físicas [14] o nuevos medicamentos farmacéuticos [15].

Aunque la mayor parte de la teoría existente detrás de las redes neuronales se conoce desde hace tiempo, no ha sido posible explotar el máximo de sus posibilidades hasta contar con suficiente capacidad de proceso mediante el uso de unidades de procesamiento gráfico (GPU). Esto, unido a la gran disponibilidad de datos para procesar y directamente influir en la toma de decisiones, conocido como Big data y detallado en [16], ha producido que en los últimos años se vuelva la vista atrás hacia las redes neuronales artificiales.

El deep learning es una tecnología de aprendizaje automático, basado en las redes neuronales artificiales, que permite apilar capas simples que apli-
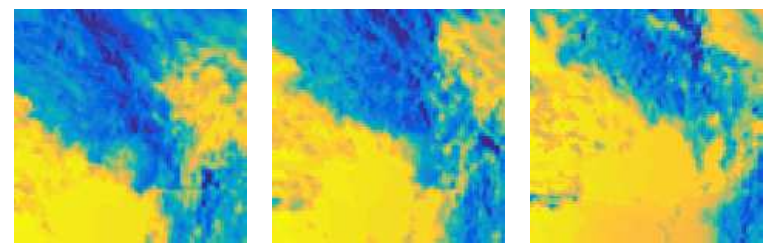

Figura 4: Evolución horaria de los datos de irradiancia en las imágenes por satélite.

can transformaciones no lineales a los datos de entrada al problema. Utilizando esta composición de transformaciones es posible aprender funciones muy complejas de los datos y obtener una representación más abstracta de los mismos.

Dicho de otra forma, las técnicas de deep learning aprenden a resolver problemas muy complejos generalizando a partir de ejemplos proporcionados a la red neuronal utilizando optimizadores generalmente basados en las técnicas de gradiente descendiente y backpropagation. Es por esto que resulta necesario diseñar una estructura de la red neuronal capaz de modelar adecuadamente el problema, cuyos parámetros se ajustarán teniendo en cuenta una función a minimizar para los datos de entrenamiento que se introduzcan. Esto es un proceso iterativo que normalmente requiere de horas, días, e incluso semanas de entrenamiento dependiendo de la complejidad del problema, hasta alcanzar un estado que permita resolver el problema para nuevas instancias de manera solvente.

En este trabajo se presenta el uso de Deep learning para predecir cuál será la irradiancia en una ubicación determinada con una hora de antelación a partir de una entrada de imágenes de satélite y de la irradiancia esperada fuera de la atmósfera para dicha ubicación en ese momento.

\subsection{DATOS DE ENTRADA}

Tal y como se ha comentado previamente, existen varios servicios capaces de calcular la irradiancia en cualquier ubicación y momento a partir de imágenes de satélite. Además, utilizando una serie de imágenes consecutivas centradas en un mismo punto, puede inferirse el movimiento de las nubes y, por tanto, sería posible predecir su próximo estado como puede verse en la figura 4.

Teniendo en cuenta esto, y la gran capacidad de las redes neuronales a la hora de procesar imágenes y extraer características de las mismas, la principal entrada a la red neuronal será la serie de imágenes inmediatamente anteriores al momento de predicción. Dada su mayor disponibilidad y precisión tal y como se demuestra en la sección 3, los datos utilizados son los de MSGCPP. 
Aunque estos datos contienen mucha información sobre la evolución de las nubes en los alrededores de la zona de interés, carecen de información de la salida esperada en una situación ideal. Por ello, además de la entrada de satélite se incluye la curva de irradiancia esperada según la hora y fecha. Este es un valor que puede calcularse geométricamente a partir de estos datos y sirve para guiar la predicción que deberá ser ajustada por la red neuronal teniendo en cuenta la situación actual de la atmósfera.

Ambas entradas han sido ajustadas y sincronizadas para asegurar que los datos de entrada son correctos y la red neuronal es capaz por tanto de predecir datos futuros. Para entrenar la red neuronal se han utilizado datos de irradiancia centrados en la Universitat Jaume I y se ha tratado de predecir el dato de irradiancia proporcionado por el mismo satélite con una hora de antelación. El conjunto de datos se ha dividido en tres subconjuntos:

- Entrenamiento: Datos correspondientes al año 2016 utilizados para entrenar la red neuronal y obtener un modelo capaz de predecir la irradiancia. En cada iteración los datos son mezclados y reintroducidos, de forma que la red no aprenda estructuras artificiales creadas por la secuencia de datos inicial.

- Test: Datos del mes de agosto de 2015 para detectar posibles sobreentrenamientos. Estos datos se evalúan a intervalos regulares durante el entrenamiento y, si el error de este conjunto crece sostenidamente, esto supone un indicador de que el modelo de la red neuronal está aprendiendo detalles del conjunto de entrenamiento que no sirven para un modelo general.

- Validación: Datos del año 2015 para medir el error de predicción del modelo ya entrenado.

\subsection{ESTRUCTURA DE LA RED}

Para el entrenamiento de la red neuronal se han considerado diferentes arquitecturas capaces de resolver el sistema. Finalmente, la red propuesta se compone de capas convolucionales para la extracción de características en las imágenes y capas densamente conectadas que procesan estas características hasta obtener una predicción de irradiancia.

Las capas convolucionales realizan operaciones en la imagen afectando únicamente a píxeles cercanos al procesado de manera invariante a la posición. De esta forma, es posible detectar formas u objetos independientemente de su posición. Esto permite extraer características de las nubes en diferentes imágenes y predecir su evolución.

Por otra parte, las capas densamente conectadas permiten hacer un análisis más global de la situación e inferir un resultado a partir del mismo. No obstante, este tipo de capas son más susceptibles a sobreentrenarse aprendiendo detalles del conjunto de entrenamiento que no son útiles para nuevas instancias del problema. Por tanto, su número de neuronas se ha mantenido bajo para evitar este tipo de situaciones.

Como función a minimizar se ha utilizado el error cuadrático medio (MSE), siendo una medida que indica la diferencia entre la predicción realizada y la esperada. Sin embargo, como medida de evaluación se ha utilizado el RMSE separado en las dos componentes ya definidas en (1). Esta medida permite mostrar de una forma más visual el error pero no aporta más información a la hora de entrenar y es más compleja computacionalmente.

\section{RESULTADOS}

Los resultados obtenidos con la red neuronal han sido comparados con dos modelos de predicción sencillos que se encuentran comúnmente en la bibliografía: modelo persistente, usado en [17] y [18], y modelo de coeficiente de nubosidad, usado en [19] y [20].

El modelo persistente asume que las condiciones climatológicas en el último instante disponible se mantienen para el instante de predicción. Por tanto, utilizando un modelo de cielo despejado se calcula la proporción respecto a la última medición y se aplica este mismo coeficiente para el instante de predicción. De esta forma, en días en que las condiciones no varían demasiado, la predicción es bastante precisa.

Por otra parte, el modelo de coeficiente de nubosidad asume que una única medida para calcular el coeficiente a aplicar en el instante de predicción está sujeta a ruido y variaciones rápidas. Por tanto, hace la media de las últimas medidas disponibles filtrando el posible ruido.

Ninguno de estos dos modelos es perfecto ya que fallan en días nubosos y, por tanto, de condiciones cambiantes. Sin embargo, en un clima mayoritariamente soleado como el propuesto, producen resultados razonablemente buenos y son de este modo buenos modelos con los que compararse.

Aunque las predicciones pueden compararse directamente como en la figura 5, es difícil apreciar diferencias entre los métodos propuestos. En esta figura se muestran tres días, uno soleado y dos parcialmente nublados, y los diferentes métodos 

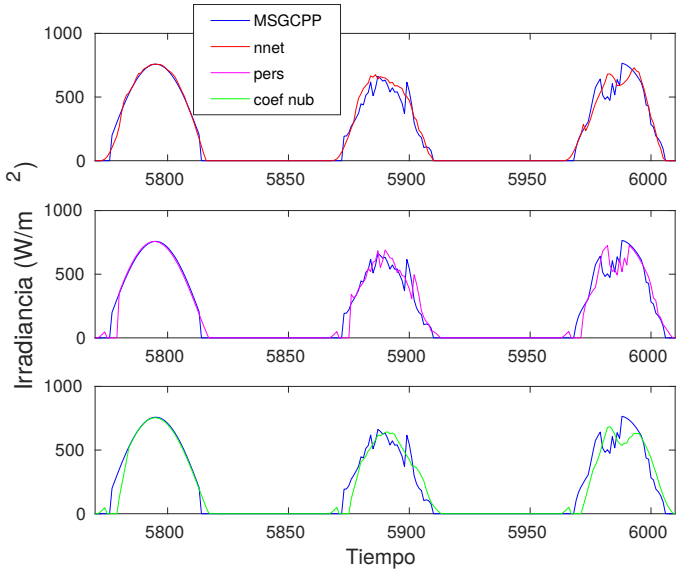

Figura 5: Comparación de los modelos de predicción de irradiancia para tres días.

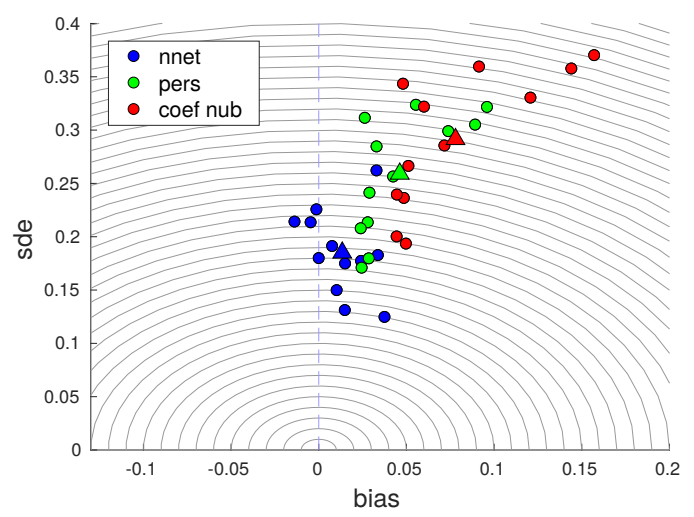

Figura 6: Resultados de los modelos de predicción para una hora con los datos de la UJI año 2015. Con puntos se representa la media mensual de cada modelo, y con triángulos la anual para los dos ejes: error sistemático (bias) y desviación estándar (sde).

junto con los valores que se intentan predecir. Se puede apreciar que todos los métodos son capaces de predecir con gran precisión días soleados pero no ocurre lo mismo con los días nublados donde cometen errores significativos.

Por ello, al igual que en el estudio de los modelos de estimación a partir de datos de satélite, los resultados han sido representados en un gráfico con dos ejes, bias y desviación estándar, para la media de cada mes del año evaluado. La figura 6 muestra los resultados para los modelos de predicción comparados de esta forma, donde se pueden apreciar de una manera mucho más clara las diferencias.

Como se puede observar en esta figura, la red neuronal, representada en azul, produce los mejores resultados tanto en error sistemático como

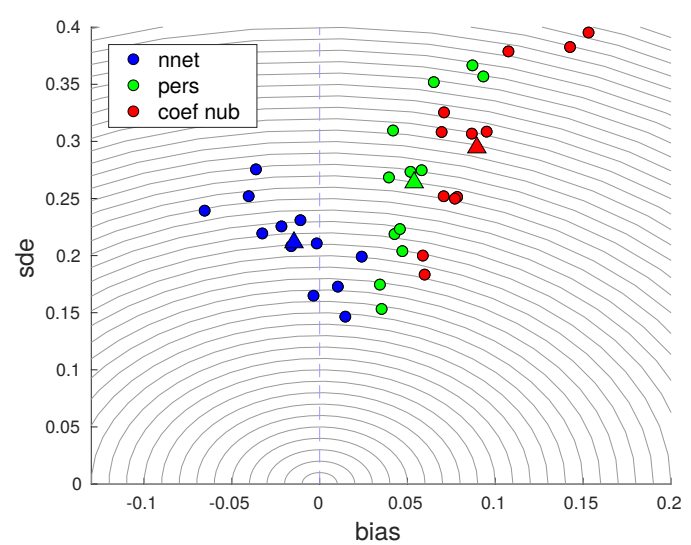

Figura 7: Resultados de los modelos de predicción para una hora con los datos de Marinha da Ondas año 2015. Con puntos se representa la media mensual de cada modelo, y con triángulos la anual para los dos ejes: error sistemático (bias) y desviación estándar (sde).

en desviación estándar. La media anual, mostrada con un triángulo en lugar del circulo que representa la mensual, se encuentrá próxima al 0 de error sistemático y alrededor de 0.18 de desviación estándar. El modelo persistente, dibujado en verde, produce mejores resultados que el de coeficiente de nubosidad ya que la mayoría de días son soleados y por tanto una única medición es suficiente para estimar la claridad del día y de este modo la irradiancia.

Los modelos simples tienen una desviación sistemática positiva causada por la necesidad de estimar un valor al comienzo del día cuando aún no se dispone de ninguna medida. En estos casos se estima un día próximo al ideal, que en muchas ocasiones no resulta ser cierto, y por tanto la media acaba estando desviada positivamente. Esto es aún mas notable en el modelo de coeficiente de nubosidad.

Otro resultado positivo de la red neuronal es que produce resultados mas fiables a lo largo del año, las medias mensuales se encuentran más cercanas, evidenciando que los resultados son más consistentes. La causa de este comportamiento es que los modelos de predicción simples se basan en que las condiciones del día se mantengan estables, cosa que no siempre ocurre, en consecuencia los resultados de predicción de meses donde no se cumple están muy desviados de la media.

En cuanto a la generalización de la red neuronal, en la figura 7 se presentan los resultados de predicción para una localización en la que la red neuronal no se ha entrenado. Es decir, además de ser datos de un año diferente como el caso anterior, 
son datos de una localización diferente. En este caso la red neuronal no puede conocer detalles de la entrada que le ayuden a la hora de predecir, por ejemplo componentes de viento habituales o especificidades geográficas.

Estos resultados muestran que la red obtiene resultados muy similares en media, aunque un poco menos consistentes. La red neuronal sigue siendo el mejor modelo de predicción de los comparados, siendo capaz de predecir radiación razonablemente bien en un lugar en el que no ha sido entrenada. En concreto, la red alcanza un error sistematico cercano al -0.01 de bias y 0.20 de desviación estándar.

Queda demostrada por tanto la capacidad de generalizar de la red neuronal, cuyos resultados podrían mejorarse reentrenando con datos de otros años de la misma localización, pero que aún así produce una previsión más precisa.

\section{CONCLUSIONES}

En este trabajo se han estudiado dos de los métodos existentes para la estimación de la irradiancia solar sobre la superfície terrestre a partir de datos de satélite con una granularidad de 15 minutos. Se han comparado ambos métodos con mediciones reales de un piranómetro, obteniéndose un mejor resultado con el servicio MSGCPP, basado en el algoritmo Cloud Physical Properties.

Además, se ha propuesto una red neuronal artifical para la predicción de la irradiancia en una ubicación determinada con una hora de antelación. Por último, se ha analizado la efectividad de dicha predicción para datos de validación no utilizados en el entrenamiento (en un año y localización diferentes). Con el modelo de predicción propuesto se ha obtenido una medida de error significativamente menor que la observada para métodos de predicción más sencillos utilizados en otras aplicaciones.

\section{Agradecimientos}

Este trabajo ha sido parcialmente realizado bajo los proyectos "Dimensionado y uso óptimo de sistemas de almacenamiento de energía en microrredes y redes de distribución inteligentes"(P1·1B2015-36) e "Integración de fuentes de energía renovables y control de flujos de potencia en redes HVDC mediante convertidores modulares multi-nivel"(DPI2014-53245-R) financiados, respectivamente, por la Universitat Jaume I de Castellón y el Ministerio de Economía junto con los fondos europeos FEDER.

\section{Referencias}

[1] Secretariat REN21, "Renewables 2016 global status report," REN21, Paris, Tech. Rep, 2016.

[2] A. Jäger-Waldau, "Pv status report 2016," JRC Science for Policy Report (Publications Office of the European Union, 2016), 2016.

[3] F. Katiraei and J. R. Aguero, "Solar pv integration challenges," IEEE Power and Energy Magazine, vol. 9, no. 3, pp. 62-71, 2011.

[4] C. Palmieri, "Energy storage in abu dhabi and dubai - a closer look," EES International - The Electrical Energy Storage Magazine, 2016.

[5] M. M. Thackeray, C. Wolverton, and E. D. Isaacs, "Electrical energy storage for transportation-approaching the limits of, and going beyond, lithium-ion batteries," Energy ES Environmental Science, vol. 5, no. 7, pp. 7854-7863, 2012.

[6] B. McCloskey, D. Bethune, R. Shelby, T. Mori, R. Scheffler, A. Speidel, M. Sherwood, and A. Luntz, "Limitations in rechargeability of li-o2 batteries and possible origins," The journal of physical chemistry letters, vol. 3, no. 20 , pp. 3043-3047, 2012.

[7] G. Girishkumar, B. McCloskey, A. Luntz, S. Swanson, and W. Wilcke, "Lithium- air battery: promise and challenges," The Journal of Physical Chemistry Letters, vol. 1, no. 14, pp. 2193-2203, 2010.

[8] H. Beltran, J. Barahona, R. Vidal, J. Alfonso, C. Ariño, and E. Pérez, "Ageing of different types of batteries when enabling a pv power plant to enter electricity markets," in Industrial Electronics Society, IECON 201642nd Annual Conference of the IEEE. IEEE, 2016, pp. 1986-1991.

[9] W. Greuell, J. F. Meirink, and P. Wang, "Retrieval and validation of global, direct, and diffuse irradiance derived from SEVIRI satellite observations," Journal of Geophysical Research Atmospheres, vol. 118, no. 5, pp. 2340-2361, 2013.

[10] B. Espinar, C. Hoyer-Klick, M. Lefèvre, M. Schroedter-Homscheidt, and L. Wald, "USER 'S GUIDE to the MACC-RAD Services on solar energy radiation resources March 2015," no. March, 2015. 
[11] A. Woyte, K. D. Brabandere, B. Sarr, and M. Richter, "The quality of satellite-based irradiation data for operations and asset management," in 32nd European Photovoltaic Solar Energy Conference and Exhibition THE, 2016, pp. 1470-1474.

[12] Y. LeCun, Y. Bengio, and G. Hinton, "Deep learning," Nature, vol. 521, no. 7553, pp. 436444, 2015.

[13] K. Simonyan and A. Zisserman, "Very deep convolutional networks for largescale image recognition," arXiv preprint arXiv:1409.1556, 2014.

[14] P. Baldi, P. Sadowski, and D. Whiteson, "Searching for exotic particles in high-energy physics with deep learning," Nature communications, vol. 5, 2014.

[15] J. Ma, R. P. Sheridan, A. Liaw, G. E. Dahl, and V. Svetnik, "Deep neural nets as a method for quantitative structure-activity relationships," Journal of chemical information and modeling, vol. 55, no. 2, pp. 263-274, 2015.

[16] A. McAfee, E. Brynjolfsson, T. H. Davenport, D. Patil, and D. Barton, "Big data," The management revolution. Harvard Bus Rev, vol. 90, no. 10, pp. 61-67, 2012.

[17] E. Lorenz and D. Heinemann, "Prediction of solar irradiance and photovoltaic power," Comprehensive Renewable Energy, vol. 1, pp. 239-292, 2012.

[18] L. M. Aguiar, B. Pereira, M. David, F. Diaz, and P. Lauret, "Use of satellite data to improve solar radiation forecasting with bayesian artificial neural networks," Solar Energy, vol. 122, pp. 1309-1324, 2015.

[19] H. Beltran, E. Perez, N. Aparicio, and P. Rodriguez, "Daily solar energy estimation for minimizing energy storage requirements in pv power plants," IEEE Transactions on Sustainable Energy, vol. 4, no. 2, pp. 474-481, 2013.

[20] E. Perez, H. Beltran, N. Aparicio, and P. Rodriguez, "Predictive power control for pv plants with energy storage," IEEE Transactions on Sustainable Energy, vol. 4, no. 2, pp. 482-490, 2013. 